\title{
Erratum to: Graph-based deformable matching of 3D line segments with application in protein fitting
}

Hang Dou ${ }^{1}$ - Matthew L. Baker ${ }^{2}$ - Tao Ju'

Published online: 7 July 2015

(C) Springer-Verlag Berlin Heidelberg 2015

Erratum to: Vis Comput (2015) 31:967-977

DOI 10.1007/s00371-015-1115-x

The title of the original article contains an error.

The correct title is given above.

The online version of the original article can be found under doi:10.1007/s00371-015-1115-x.

$凶$ Hang Dou

hangdou@gmail.com

1 Washington University in St. Louis, St. Louis, USA

2 Baylor College of Medicine, Houston, USA 